$\mathrm{E}$

EVALUAR
2020, Vol. 20, No. 2

ISSN $1667-4545$

Recuperado de https://revistas.unc.edu.ar/index.php/revaluar

Laboratorio de Evaluación Psicológica y Educativa

Facultad de Psicología - Universidad Nacional de Córdoba

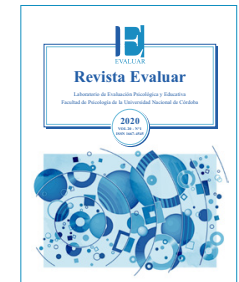

\title{
Diseño y validación de una escala de actitudes hacia las matemáticas
}

\section{Design and Validation of an Attitudes towards Mathematics Scale}

\author{
Florencia Stelzer $^{1,2 *}$, Santiago Vernucci ${ }^{1,2}$, Yesica Aydmune ${ }^{1,2}$, \\ Macarena del Valle ${ }^{1,2}$, María Laura Andrés ${ }^{1,2}$ \\ 1 - Consejo Nacional de Investigaciones Cientificas y Técnicas (CONICET). \\ 2 - Facultad de Psicología. Universidad Nacional de Mar del Plata (UNMDP), Argentina.
}

\author{
Introducción \\ Método \\ Resultados \\ Discusión \\ Referencias
}

Recibido: 29/01/2020 Revisado: 10/03/2020 Aceptado: 02/04/2020

\section{Resumen}

El objetivo de este estudio es evaluar las propiedades psicométricas de una Escala de Actitudes hacia las Matemáticas (EAM) para estudiantes que finalizan la escolaridad primaria e inician la secundaria. Para ello, se diseñó una escala y se evaluaron sus propiedades psicométricas y su capacidad predictiva. Participaron 193 estudiantes de sexto grado de primaria y primer y segundo año de secundaria (edad: $\mathrm{ME}=12.7$ años; $\mathrm{DE}=0.36$ ). Las correlaciones ítems-total y el alfa de Cronbach $(\alpha=.92)$ indicaron que la escala es consistente. El análisis factorial exploratorio indicó una estructura de tres factores, designados competencia percibida, gusto/motivación por las matemáticas y conductas de compromiso escolar. Se observaron correlaciones moderadas entre las tres dimensiones de la EAM y medidas de éxito y productividad académica en matemáticas. Estos resultados sugieren que la EAM representa un medio confiable y válido para la estimación de las actitudes hacia las matemáticas.

Palabras clave: actitud hacia las matemáticas, medición, propiedades psicométricas, escolares

\section{Summary}

The aim of this study is to evaluate the psychometric properties of an Attitudes towards Mathematics Scale (AMS) for students who are finishing elementary school and starting high school. For this purpose, a specific measure was designed, and its psychometric properties and predictive capacity were evaluated. 193 students of elementary (sixth grade) and high school (first and second year) participated in the study $(\mathrm{M}$ age $=12.7$ years, $\mathrm{SD}=0.36)$. Item-total correlations and Cronbach's alpha $(\alpha=.92)$ indicated that the scale is consistent for construct measurement. Exploratory factor analysis showed a three-factor structure, named perceived competence, liking/motivation for mathematics, and school engagement behaviours. Regarding predictive validity, moderate correlations were found among scale's dimensions and measures of academic productivity and success in mathematics. These results suggest that the AMS represents a reliable and valid tool for assessing attitudes towards mathematics.

Keywords: attitudes towards mathematics, assessment, psychometric properties, students

*Correspondencia a: Florencia Stelzer, Dean Funes 3200 - 3 V / 7600 - Mar del Plata. Te.: +54 223 4475696. E-mail: florenciastelzer@gmail.com Cómo citar este artículo: Stelzer, F., Vernucci, S., Aydmune, Y., del Valle, M., Andrés, M. L. (2020). Diseño y validación de una escala de actitudes hacia las matemáticas. Revista Evaluar, 20(2), 51-68. Recuperado de https://revistas.unc.edu.ar/index.php/revaluar 


\section{Introducción}

Diferentes estudios indicaron que las actitudes que los estudiantes muestran hacia las matemáticas y su aprendizaje predicen su desempeño en esta área del conocimiento (Aiken \& Dreger, 1961; Burrus \& Moore, 2016; Chen et al., 2018; Miñano \& Castejón, 2011; Miranda, 2012; Pitsia, Biggart, \& Karakolidis, 2017). Aunque existen diversas definiciones del término actitud, estas coinciden en caracterizarla como una tendencia evaluativa hacia un objeto y sus símbolos, que se construye principalmente a través de la experiencia. En este sentido, las actitudes son objeto de estudio de la Psicología (originalmente de la Psicología Social), pero también resultan de interés para diversos profesionales en distintos ámbitos de trabajo, como aquellos involucrados en la enseñanza-aprendizaje de las matemáticas (Auzmendi, 1992; Farías-Mata, 2015; Muñoz-Cantero \& Mato-Vázquez, 2008; Vázquez-Alonso \& Manassero-Mas, 1995; Zanna \& Rempel, 1988). En el ámbito de la educación matemática predominan las definiciones multidimensionales del constructo. Farías-Mata (2011), por ejemplo, caracteriza las actitudes hacia las matemáticas como "tendencias psicológicas evaluativas, inferidas a partir de ciertas respuestas cognoscitivas, afectivas y conductuales, que expresan en conjunto algún grado de aprobación o desaprobación hacia las matemáticas" (p. 75). En consonancia con esta definición, distintos autores han considerado tres dimensiones de las actitudes hacia las matemáticas: cognitiva, afectiva y comportamental ( $\mathrm{p}$. ej., Auzmendi, 1992; Eagly \& Chaiken, 2005; Pedrosa, Astiz, Montero, \& Todisco, 2016). La dimensión cognitiva se refiere al conjunto de representaciones o creencias sobre las matemáticas; la afectiva considera la carga emocional relacionada con dichas representaciones; la comportamental estima las intenciones y tendencias de conducta en relación con las matemáticas y su aprendizaje. En estas dimensiones se han propuesto diferentes aspectos o subdimensiones; no obstante, se observan diferencias entre los autores respecto de cuáles deberían integrar cada constructo.

En lo referido a la dimensión cognitiva, diferentes estudios (Adelson \& McCoach, 2011; Auzmendi, 1992; González-Pienda et al., 2012; Palacios, Arias, \& Arias, 2014; Tapia \& Marsh, 2004) indicaron que la competencia percibida podría representar un subdimensión. También ha sido llamada confianza en sí mismo o autopercepción matemática y es definida como el autoconcepto que los estudiantes poseen respecto a su desempeño y capacidad de aprendizaje de las matemáticas (Adelson \& McCoach, 2011; Fennema \& Sherman, 1976; Tapia \& Marsh, 2004).

Otra subdimensión cognitiva podría ser la utilidad o valor percibido (Adelson \& McCoach, 2011; Auzmendi, 1992; Cueli, González-Castro, Álvarez, García, \& González-Pienda, 2014; Eccles \& Wigfield, 2002; Fennema \& Sherman, 1976; Palacios et al., 2014; Tapia \& Marsh, 2004), la cual es definida como el grado en el que los estudiantes perciben que las matemáticas resultan útiles para sus objetivos actuales y futuros en el ámbito escolar, laboral y en la vida diaria (Adelson \& McCoach, 2011; Fennema \& Sherman, 1976; Tapia \& Marsh, 2004). Esta subdimensión se relacionaría con la motivación extrínseca, esto es, la energización o activación de las conductas producto de las consecuencias que su ejecución implica (Ryan \& Deci, 2000). Si bien la mayor parte de las investigaciones sugieren que la utilidad percibida representa una dimensión independiente de las actitudes hacia las matemáticas (Eccles \& Wigfield, 1995; Eccles, Wigfield, Harold, \& Blumenfeld, 1993; Palacios et al., 2014; Tapia \& Marsh, 2004), algunos estudios aportaron evidencia en un sentido contrario. Por ejemplo, Adelson y McCoach (2011) hallaron que las va- 
riaciones en los indicadores de este componente no se distinguían con claridad respecto de las variaciones en indicadores que describían el gusto por las matemáticas y la autopercepción matemática. Asimismo, Muñoz-Cantero y Mato-Vázquez $(2006,2008)$ informaron que los indicadores típicamente utilizados para medir el valor percibido y el gusto por las matemáticas se agrupaban en un único factor llamado agrado y utilidad de las matemáticas en un futuro.

Respecto de la dimensión afectiva, una subdimensión propuesta por diversos investigadores (Adelson \& McCoach, 2011; Aiken, 1974, 1979; Aiken \& Dreger, 1961; Auzmendi, 1992; Fennema \& Sherman, 1976; Hurtado-Mondoñedo, 2011; Tapia \& Marsh, 2004) es el disfrute o gusto por las matemáticas, el cual se refiere al grado en el que los estudiantes experimentan placer o disfrute, tanto al realizar actividades que involucran a las matemáticas como durante el aprendizaje de este conocimiento. Ciertos autores (p. ej., González-Pienda et al., 2012) incluyeron al disfrute o gusto por las matemáticas dentro de otra dimensión afectiva más general, tal como la motivación intrínseca. Esto se debe a que el disfrute en la realización de tareas matemáticas constituye un aspecto de la motivación intrínseca (Stipek, 2002), la cual se relaciona con a la energización o impulso que activa los comportamientos producto del placer derivado de su ejecución (Deci \& Ryan, 1985, 2000; Ryan \& Deci, 2000). No obstante, otros autores (Tapia \& Marsh, 2004) han aportado evidencia a favor de una distinción entre el gusto por las matemáticas y la motivación, entendida esta última como el interés en las matemáticas y el deseo de continuar su estudio.

Otra subdimensión afectiva es la ansiedad matemática (Aiken 1979; Aiken \& Dreger, 1961; Auzmendi, 1992; Fennema \& Sherman, 1976; González-Pienda et al., 2012; Hurtado-Mondoñedo, 2011), la cual se entiende como el grado en que los estudiantes experimentan sentimientos de ansiedad, nerviosismo o temor al realizar actividades matemáticas (Fennema \& Sherman, 1976; Suinn \& Edwards, 1982). Aunque algunos autores (Auzmendi, 1992; Hurtado-Mondoñedo, 2011) informaron que los indicadores de ansiedad se agrupaban en un factor diferente respecto de los que describían la confianza en el propio desempeño en matemática (competencia percibida), otros estudios (Melancon, Thompson, \& Becnel, 1994; Mulhern \& Rae, 1998; O’Neal, Ernest, McLean, \& Templeton, 1988; Tapia \& Marsh, 2004) señalaron que los indicadores de ambos aspectos tendían a agruparse en un mismo factor.

En lo relativo a la dimensión comportamental, en la literatura no se ha analizado aún con suficiente claridad sus subdimensiones e indicadores. En la mayor parte de los cuestionarios, los ítems que consideran la tendencia o inclinación a actuar respecto de las matemáticas son escasos, y han sido definidos como indicadores de dimensiones del componente afectivo (p. ej., Puedo pasarme horas estudiando matemáticas y haciendo ejercicios como indicador de la dimensión gusto por las matemáticas [Palacios et al., 2014]; Me gustaría poder evitar utilizar las matemáticas en la universidad como indicador de la dimensión motivación [Tapia \& Marsh, 2004]). Recientemente, Pedrosa et al. (2016) propusieron evaluar al componente comportamental como una dimensión independiente de las actitudes hacia las matemáticas y desarrollaron indicadores que se refieren tanto a la predisposición conductual como a las formas efectivas de actuación. Tales indicadores describen la dedicación de tiempo y esfuerzo en actividades de matemáticas (p. ej., Preparo con tiempo suficiente los exámenes de matemáticas), así como la declaración de preferencias (p. ej., Prefiero estudiar cualquier otra materia antes que estudiar matemáticas). Sin embargo, también se han establecido indicadores 
que apuntan a juicios de valor sobre esta asignatura (p. ej., Las matemáticas me resultan útiles para entender las demás áreas; Las matemáticas son valiosas y necesarias), por lo que la diferenciación conceptual y operacional respecto de las dimensiones cognitiva y afectiva de las actitudes no resulta totalmente clara. Además, la tendencia a actuar y la actuación efectiva de los sujetos se relacionan con esquemas conceptuales diferentes. La primera se encuadra dentro de los modelos teóricos de actitudes hacia las matemáticas, en la medida en que la actitud comprende una tendencia evaluativa que puede concretarse o no en una acción dependiendo de condiciones contextuales (Auzmendi, 1992; Pedrosa et al., 2016). Por el contrario, la actuación efectiva de los sujetos ha sido considerada en mayor medida como un indicador del comportamiento de compromiso escolar ante esta asignatura (Fredricks, Blumenfeld, \& Paris, 2004; Fredricks et al., 2016).

Si bien se han diseñado instrumentos de medición de las actitudes hacia las matemáticas en sujetos hispanohablantes, la mayor parte de estos son aplicables a estudiantes de nivel secundario y universitario, y su confiabilidad y validez ha sido contrastada principalmente en muestras españolas (p. ej., Alemany-Arrebola \& Lara, 2010; Auzmendi, 1992; Bazán \& Sotero, 1998; Muñoz-Cantero \& Mato-Vázquez, 2008; Vallejo-Seco \& Escudero-García, 1999; para una excepción en estudiantes de nivel inicial y primario ver Román-Alegre, Mera-Cantillo, Aragón-Mendizábal, \& Delgado, 2019; González-Pienda et al., 2012; Palacios et al., 2014). En Latinoamérica, en general se han utilizado y validado las escalas desarrolladas en España y Estados Unidos, siendo la propuesta por Auzmendi (1992) la más utilizada en estudiantes de nivel terciario y universitario (p. ej., Escalera-Chávez, Moreno-García, \& Rojas-Kramer, 2019; Dörfer \& Ulloa-Duque, 2016; Cardoso-Espinosa, Vanegas-López, \& Cerece-
do-Mercado, 2012; Petriz-Mayen, Barona-Ríos, Lopez-Villarreal, \& Quiroz-González, 2010) y la de Fennema y Sherman (1976) en participantes de nivel secundario (p. ej., Meza-Cascante et al., 2019). En el contexto específico de Argentina, los instrumentos válidos y confiables son limitados y han sido propuestos principalmente para estudiantes de nivel universitario (p. ej., Abal, Auné, \& Attorresi, 2018) o del segundo ciclo del nivel secundario (p. ej., Pedrosa et al., 2016).

Ahora bien, la adquisición de conocimientos y el desarrollo de habilidades en matemáticas resultan imprescindibles para el funcionamiento adaptativo en la sociedad actual (Butterworth, 2005). Al respecto, diferentes informes indicaron que un porcentaje notorio de estudiantes de Argentina presenta dificultades en el aprendizaje de las matemáticas. Por ejemplo, los resultados de los últimos dos operativos nacionales de evaluación de los logros de aprendizaje correspondientes al nivel primario indicaron que más de un $40 \%$ de los niños egresa sin adquirir los conocimientos esperados (Ministerio de Educación y Deportes, 2017; Ministerio de Educación, Cultura, Ciencia y Tecnología, 2019). En un sentido semejante, los resultados del estudio PISA 2018 indicaron que el $69 \%$ de los estudiantes de 15 y 16 años de Argentina se ubicaban dentro de los dos niveles más bajos de desempeño, es decir, no alcanzaban el nivel de competencias básicas en matemáticas que es necesario para desenvolverse funcionalmente en la sociedad moderna (Organisation for Economic Cooperation and Development, 2019).

Diferentes estudios indican que las actitudes hacia las matemáticas se relacionan con el desempeño y aprendizaje de esta asignatura (p. ej., Abu-Hilal, 2000; Gómez-Chacón, 2009; Hemmings, Grootenboer, \& Kay, 2011), por lo que disponer de instrumentos válidos y confiables que permitan la medición de las actitudes hacia las matemáticas resulta fundamental. Además, 
dado que es sabido que la actitud hacia las matemáticas se torna más negativa mientras mayor es la edad de los estudiantes (Núñez, et al., 2005; Watt, 2000), es de relevancia contar con medidas adecuadas para los diferentes niveles de formación educativa, y así poder realizar evaluaciones y seguimientos sobre la relación de los alumnos con la asignatura a lo largo del tiempo y realizar intervenciones pertinentes si fueran necesarias (Cubillo \& Ortega, 2000).

El acceso a un instrumento válido y confiable de medición de las actitudes hacia las matemáticas en alumnos argentinos que finalizan la escolaridad primaria e inician la secundaria permitiría analizar el estado en que se encuentra este fenómeno en esta población. Además, una indagación diagnóstica sobre la actitud actual de los estudiantes hacia las matemáticas puede facilitar a futuro la puesta en marcha de intervenciones que contribuyan a su éxito académico en esta disciplina (Guvercin \& Verbovskiy, 2014; Pere1s, Dignath, \& Schmitz, 2009; Qaisar, Dilshad, \& Butt, 2015). Por lo anterior, el objetivo de este trabajo fue desarrollar una escala para la medición de las actitudes hacia las matemáticas en estudiantes que finalizan la escolaridad primaria e inician la escolaridad secundaria de Argentina y aportar evidencias sobre su confiabilidad y validez predictiva. La escala fue llamada Escala de Actitudes hacia las Matemáticas (EAM).

\section{Metodología}

Tipo de estudio y diseño

Se efectuó un estudio instrumental (Montero \& León, 2005). Se diseñó la Escala de Actitudes hacia las Matemáticas (EAM). Para ello, en función de la revisión de la literatura, se con- sideraron tres dimensiones de las actitudes hacia las matemáticas: (a) gusto/motivación por las matemáticas, que considera el grado en el que los estudiantes manifiestan placer o disfrute al realizar actividades matemáticas o destinadas a su aprendizaje (Adelson \& McCoach, 2011; Tapia \& Marsh, 2004), así como la energización y orientación del comportamiento hacia este tipo de actividades (González-Pienda et al., 2012); (b) competencia percibida, que se refiere al autoconcepto que los estudiantes poseen como aprendices de matemáticas, incluyendo las creencias respecto a su desempeño en matemáticas y su capacidad de aprendizaje (Adelson \& McCoach, 2011; Tapia \& Marsh, 2004); y (c) conductas de compromiso escolar, que involucran aquellos comportamientos específicos que evidencian el esfuerzo y la dedicación que los estudiantes invierten en el aprendizaje de las matemáticas. Esta dimensión incluye indicadores que estiman la atención sobre las instrucciones y explicaciones del docente, la planificación del tiempo destinado al estudio de esta asignatura, la realización de tareas escolares en el hogar y de actividades en clase, el interés por su correcta concreción y la preparación de los materiales necesarios para la clase.

Estas dimensiones fueron seleccionadas debido a que reflejan los tres componentes del constructo, a saber, cognitivo, afectivo y comportamental. Además, para el caso de las dos primeras, diferentes estudios han aportado evidencia a favor de considerarlas como dimensiones de la actitud hacia las matemáticas (Adelson \& McCoach, 2011; Auzmendi, 1992; González-Pienda et al., 2012; Palacios et al., 2014; Tapia \& Marsh, 2004). Respecto de la dimensión de conductas de compromiso escolar, si bien dentro de los modelos teóricos de actitudes hacia las matemáticas no se la ha considerado, existe evidencia que indica que la conducta específica que los sujetos manifiestan respecto a las matemáticas y su aprendiza- 
je contribuye de forma diferencial al desempeño y su aprendizaje (Fredricks et al., 2004; Hattie, 2009; Marsh et al., 2016), por lo que su medición reviste interés.

Para la selección de los ítems que integran el cuestionario se revisó el contenido de los cuestionarios en inglés y español más utilizados para la medición de este constructo (p. ej., Adelson \& McCoach, 2011; Aiken, 1979; Auzmendi, 1992; Fennema \& Sherman, 1976; González-Pienda et al., 2012; Muñoz-Cantero \& Mato-Vázquez, 2008; Palacios et al., 2014; Tapia \& Marsh, 2004) y se seleccionaron aquellos que a juicio de dos expertos en la temática reflejaban con mayor precisión y claridad cada una de las dimensiones propuestas. En el caso de los ítems en inglés, fueron traducidos por un experto en la temática con dominio del idioma.

Conforme a lo sugerido para la evaluación de la conducta de sujetos de entre 11 y 15 años de edad, se optó por una escala de respuesta Likert de cuatro opciones (Chambers \& Johnston, 2002; Lozano, García-Cueto, \& Muñiz, 2008; Mellor \& Moore, 2014) que oscila entre totalmente parecido a mí y totalmente distinto a mí. Se decidió no incluir una opción de respuesta neutral a fin de propiciar la elección de una tendencia respecto de cada ítem.

La versión inicial del cuestionario estuvo integrada por 28 ítems, de los cuales 8 se refieren a la dimensión competencia percibida (5 ítems con puntuación invertida); 9 a gusto/motivación por las matemáticas intrínseca (4 ítems con puntuación invertida) y 10 a conductas de compromiso escolar (2 ítems con puntuación invertida).

\section{Participantes}

Los participantes fueron seleccionados de forma no probabilística, por disponibilidad.
La muestra inicial estuvo integrada por 193 estudiantes de sexto grado del nivel primario, y de primer y segundo año del nivel secundario (sexto grado: $\mathrm{n}=25$; primer año: $\mathrm{n}=100$; segundo año: $\mathrm{n}=68$ ), asistentes a un establecimiento educativo de gestión privada de la ciudad de Mar del Plata, Argentina. Fueron excluidos del análisis de los datos aquellos casos en los que los progenitores o responsables legales informaron la presencia de diagnóstico de trastornos mentales o neurológicos. La muestra final quedó conformada por 181 estudiantes (sexto grado: $\mathrm{n}=20$ [9 varones]; primer año: $\mathrm{n}=91$ [40 varones]; segundo año: $\mathrm{n}=$ 66 [25 varones]).

\section{Instrumentos}

Versión reducida de la escala de desempeño académico APRS (Academic Performance Rating Scale; DuPaul, Rapport, \& Perriello, 1991). La escala original está integrada por 19 ítems y constituye un cuestionario cerrado de opción múltiple que indaga la percepción del docente sobre el desempeño académico del estudiante. Para este estudio, se aplicaron únicamente los ítems que se refieren al desempeño en matemáticas (12 ítems); cinco ítems pertenecen a la subescala de éxito académico y siete ítems a la subescala de productividad académica. La primera se relaciona con los resultados observables del aprendizaje y considera tanto el rendimiento del estudiante durante el trabajo escolar como su capacidad para aprender rápidamente (p. ej., ¿Cómo es en términos generales la calidad de sus trabajos escolares?; ¿Con cuánta rapidez aprende los niños nuevos contenidos?). La subescala productividad académica considera aquellos comportamientos que son importantes para alcanzar el éxito académico, tales como la realización de las actividades en clase, el seguimiento de instrucciones y la ca- 
pacidad de trabajo autónomo (p. ej., ¿Con cuánta frecuencia este niño completa las tareas de una manera descuidada y apresurada?). Las respuestas se registran en una escala Likert que oscila de 1 a 5 (nunca o pobre a muy a menudo o excelente). Este cuestionario ha sido aplicado previamente en el contexto de Argentina y se han observado relaciones significativas con medidas estandarizadas del desempeño en matemáticas (Stelzer, Canet-Juric, Andrés, Vernucci, \& Richards, 2019). En el presente estudio, tanto la escala total como las subescalas mostraron adecuada consistencia interna (escala total: $\alpha=.81$; éxito académico: $\alpha$ $=.84$; productividad académica: $\alpha=.76$ ).

Ficha sanitaria. Constituye un cuestionario semicerrado de informe de los padres en el que se indaga la presencia en el estudiante de antecedentes o diagnóstico actual de trastornos psicológicos, psiquiátricos o neurológicos. En él, se pregunta si el estudiante presenta o presentó dificultades atencionales, académicas o de control de los impulsos y/o emociones. En caso de que se responda afirmativamente ante alguna dificultad, se debe detallar si se concurrió a algún profesional (especificando cuál) y qué diagnóstico brindó.

\section{Procedimiento y consideraciones éticas}

Para la participación en este estudio fue requerida la autorización por escrito de los padres, a través de la firma de un consentimiento informado en el que se detallaban los objetivos del estudio, los instrumentos de evaluación que serían utilizados y la posibilidad de abandonar la investigación en el momento en que lo solicitaran. Además, se especificaba que los datos recabados serían tratados y utilizados confidencialmente, conforme a la Declaración de Helsinki (World Medical Association, 2013) y en consonancia con los principios éticos y el código de conducta de los psicólogos, establecido y reformulado por la Asociación Americana de Psicología (American Psychological Association, 2010). Los estudiantes fueron informados por uno de los investigadores responsables sobre el estudio y sus alcances y se requirió su asentimiento verbal para participar.

La ficha sanitaria fue enviada a los progenitores a través del cuaderno de comunicaciones. El cuestionario EAM fue administrado de forma grupal en el salón habitual de clases de los estudiantes, por operadores entrenados, durante el primer trimestre del año escolar. El cuestionario de desempeño académico APRS fue enviado a los docentes durante el último trimestre del año escolar.

\section{Análisis de datos}

En primer lugar, se evaluó la correlación entre cada ítem y la puntuación total de la escala y se eliminaron aquellos ítems que mostraban correlaciones inferiores a .3 (Wieland, Durach, Kembro, \& Treiblmaier, 2017; Zaichkowsky, 1994). Se calculó luego el coeficiente alfa de Cronbach ( $\alpha$ ) para la escala total. En segundo lugar, para evaluar la estructura interna del instrumento, se realizó un análisis factorial exploratorio (AFE), utilizando el método de factorización de ejes principales y la extracción según autovalores $>1$. Para esto, se comprobó primero la adecuación de los datos a través del test de esfericidad de Barlett y de la medida de adecuación muestral de Kaiser-Meyer-Olkin (KMO). Se consideraron como indicadores de adecuación un valor $p<.05$ en el test de esfericidad de Barlett y valores $\mathrm{KMO}>.5$. En función de los resultados del primer AFE, se efectuó un segundo AFE a través del mismo método de factorización, pero estableciendo tres factores de extracción. Se utilizó la rotación oblimin 
Tabla 1

Matriz de configuración y matriz de estructura con comunalidades para la versión reducida de la Escala de Desempeño Académico.

\begin{tabular}{|c|c|c|c|c|c|c|c|}
\hline \multirow[b]{2}{*}{ Ítem } & \multicolumn{3}{|c|}{$\begin{array}{l}\text { Matriz de } \\
\text { configuración }\end{array}$} & \multicolumn{4}{|c|}{ Matriz de la estructura } \\
\hline & $\mathrm{CP}$ & $\mathrm{CC}$ & GM & $\mathrm{CP}$ & $\mathrm{CC}$ & GM & $h^{2}$ \\
\hline 2 Disfruto jugando juegos que tienen matemáticas. & & & .46 & .39 & .24 & .55 & .32 \\
\hline $\begin{array}{l}3 \text { Cuando sea mayor me gustaría tener un trabajo en el cual tenga que } \\
\text { usar matemáticas. }\end{array}$ & & & .67 & .38 & .21 & .70 & .49 \\
\hline 6 Las matemáticas son divertidas y entretenidas para mí. & .44 & & .57 & .66 & .19 & .74 & .69 \\
\hline $\begin{array}{l}11 \text { (INV) Espero tener que utilizar poco las matemáticas cuando } \\
\text { termine la escuela. }\end{array}$ & & & .65 & .31 & .13 & .64 & .42 \\
\hline $\begin{array}{l}\text { 14(INV) Me gustaría no tener la materia matemáticas el próximo } \\
\text { año. }\end{array}$ & & & .66 & .54 & .36 & .77 & .64 \\
\hline 16 (INV) Las matemáticas no tienen interés para mí. & & & .68 & .54 & .32 & .79 & .65 \\
\hline 18 Me gustaría aprender más temas de matemáticas. & & & .64 & .42 & .30 & .70 & .50 \\
\hline $\begin{array}{l}23 \text { (INV) Me disgusta estudiar matemáticas, incluso las partes más } \\
\text { fáciles. }\end{array}$ & & & .35 & .47 & .40 & .52 & .37 \\
\hline $\begin{array}{l}25 \text { Quiero llegar a tener un conocimiento más profundo de las } \\
\text { matemáticas. }\end{array}$ & & & .79 & .17 & .21 & .70 & .52 \\
\hline 1 Si me lo propongo puedo sacar buenas notas en matemáticas. & .50 & & & .55 & .36 & .25 & .33 \\
\hline $\begin{array}{l}5 \text { (INV) Me siento más torpe en matemáticas que la mayoría de mis } \\
\text { compañeros. }\end{array}$ & .62 & & & .66 & .36 & .32 & .45 \\
\hline 9 (INV) No entiendo las matemáticas. & .82 & & & .84 & .32 & .44 & .71 \\
\hline 13 Aprendo matemáticas fácilmente. & .80 & & & .83 & .29 & .47 & .70 \\
\hline $\begin{array}{l}20 \text { (INV) En matemáticas me cuesta trabajo entender qué tengo que } \\
\text { hacer. }\end{array}$ & .46 & & & .56 & .37 & .33 & .34 \\
\hline 22 Soy bueno resolviendo problemas de matemáticas. & .74 & & & .80 & .25 & .52 & .66 \\
\hline 24 (INV) Soy malo en matemáticas. & .91 & & & .90 & .34 & .42 & .81 \\
\hline 27 (INV) Las matemáticas son difíciles para mí. & .77 & & & .81 & .26 & .50 & .68 \\
\hline 4 Escucho muy atentamente al profesor en clase de matemáticas. & & .56 & & .29 & .59 & .26 & .36 \\
\hline $\begin{array}{l}7 \text { (INV) Me olvido cosas o elementos que necesito para la clase de } \\
\text { matemáticas. }\end{array}$ & & .64 & & .09 & .58 & .13 & .36 \\
\hline 10 Mi carpeta de matemáticas está completa. & & .79 & & .22 & .77 & .22 & .60 \\
\hline $\begin{array}{l}12 \text { Llego a los exámenes de matemática con todos los contenidos } \\
\text { bien estudiados. }\end{array}$ & & .53 & & .37 & .60 & .25 & .38 \\
\hline $\begin{array}{l}17 \text { En clases de matemáticas me esfuerzo por completar todos los } \\
\text { ejercicios. }\end{array}$ & & .49 & & .27 & .53 & .25 & .29 \\
\hline $\begin{array}{l}19 \text { (INV) En clase de matemáticas no recuerdo las cosas que mi } \\
\text { profesor me pide hacer. }\end{array}$ & & .46 & & .27 & .48 & .06 & .26 \\
\hline 21 Traigo completa la tarea de matemáticas a clase. & & .70 & & .27 & .71 & .22 & .50 \\
\hline 26 Termino todas las actividades que se dan en clase. & & .47 & & .48 & .60 & .36 & .44 \\
\hline
\end{tabular}

Nota. CP: competencia percibida; CC: conductas de compromiso escolar; GM/MI: gusto por las matemáticas / motivación intrínseca. 
directa ya que la literatura sugiere asociaciones entre las dimensiones propuestas en la EAM (p. ej., Adelson \& McCoach, 2011; Goetz, Cronjäger, Frenzel, Lüdtke, \& Hall, 2010; Goetz et al., 2012). Luego, se calcularon los coeficientes $\alpha$ para cada una de las dimensiones identificadas a través del AFE. Finalmente, se evaluó la validez predictiva de cada dimensión a través de sus relaciones con las escalas de productividad académica y éxito académico del APRS. Para esto, se consideró como puntuación en cada dimensión el promedio en el conjunto de ítems que las integraban.

\section{Resultados}

Análisis de confiabilidad

El análisis de confiabilidad general de la escala mostró un valor $\alpha$ de .91 . Se analizaron las correlaciones entre cada ítem y la puntuación total de la escala sin considerar el mismo ítem. El rango de las correlaciones resultantes fue de -.14 a .71. Con el propósito de incrementar la confiabilidad general de la escala, se eliminaron tres ítems que presentaban correlaciones inferio-

Tabla 2

Correlaciones entre los factores de la EAM.

\begin{tabular}{lll}
\hline & $\begin{array}{c}\text { Compromiso } \\
\text { escolar }\end{array}$ & \multicolumn{1}{c}{$\begin{array}{c}\text { Gusto / } \\
\text { motivación }\end{array}$} \\
\hline $\begin{array}{l}\text { Competencia } \\
\text { percibida }\end{array}$ & $.38 * *$ & $.49 * *$ \\
$\begin{array}{l}\text { Compromiso } \\
\text { escolar }\end{array}$ & & $.28 * *$ \\
\hline
\end{tabular}

Nota. ${ }^{* *} p<.001$.

res a .3 con el total de la escala (Wieland et al., 2017; Zaichkowsky, 1994). Uno de los ítems eliminados respondía a la escala competencia percibida (Resuelvo los problemas de matemáticas sin demasiado esfuerzo, $r=-.14$ ) y los otros dos pertenecían a la escala conducta de compromiso escolar (Preparo con tiempo suficiente los exámenes de matemáticas, $r=.09$; Reviso y corrijo los ejercicios que trabajamos en clases, $r=.17$ ). La versión final del cuestionario quedó integrada por 25 ítems $(\alpha=.92)$. El rango de correlaciones entre cada ítem y la escala sin considerar ese mismo ítem fue de .28 a .71.

\section{Validez de constructo}

Con el objetivo de establecer si el conjunto de 25 ítems seleccionados reflejaba la estructura teórica de tres dimensiones establecida en el cuestionario, se aplicó un AFE utilizando el método de factorización de ejes principales y la extracción según autovalores mayores que 1. El test de esfericidad de Barlett $\left(\chi_{(300)}^{2}=2041.266\right.$, $p<.001)$ y la medida de adecuación muestral de Kaiser-Meyer-Olkin (.904) indicaron que los datos eran adecuados para el análisis factorial.

Los resultados del AFE indicaron 5 factores con un autovalor mayor que 1 que explicaban el $64.17 \%$ de la varianza. Si bien la regla de Kaiser-Guttman sugeriría retenerlos, el cuarto y quinto factor mostraron autovalores levemente mayores que $1(<1.1)$ y cada uno explicaba menos del $4 \%$ de la varianza. Considerando que la literatura sugiere que el método de selección por la regla de Kaiser-Guttman puede conducir a la sobre factorización (Russell, 2002), teniendo en cuenta la baja proporción de varianza adicional que explica un modelo de 5 factores (7\%) y analizando el punto de inflexión en la pendiente del gráfico de sedimentación, se optó por extraer tres factores.

Se efectuó un segundo AFE a través del método de factorización de ejes principales estableciendo 3 factores de extracción. Se utilizó la rotación oblimin directa. Los tres factores obte- 
Tabla 3

Correlaciones entre las subescalas de la EAM y las subescalas del cuestionario de desempeño académico APRS.

\begin{tabular}{llll}
\hline & Gusto/motivación & Competencia percibida & Compromiso escolar \\
\hline Éxito académico & $.43 * *$ & $.54 * *$ & $.37 * *$ \\
Productividad académica & $.34 * *$ & $.41 * *$ & $.49 * *$ \\
\hline
\end{tabular}

Nota. $* p<001$.

nidos explicaban el $55.5 \%$ de la varianza. En la Tabla 1 se presenta la matriz de configuración y la matriz de la estructura con las comunalidades de cada variable.

La matriz de configuración mostró que únicamente el ítem 6 presentaba cargas superiores a .3 en más de un factor, por lo que se optó por asignarlo a aquel en el que mostraba mayor peso. El resto de los ítems presentaron cargas superiores a .3 en un único factor, al que fueron respectivamente asignados. El primer factor, integrado por 8 ítems, agrupó aquellos que describen la autopercepción de los estudiantes respecto a su desempeño en matemáticas y su capacidad de aprendizaje de esta asignatura, por lo que fue designado competencia percibida. El segundo factor, designado conductas de compromiso escolar, aunó 8 ítems que describen conductas que evidencian la planificación, inversión de tiempo y el esfuerzo destinado al aprendizaje de las matemáticas. Por último, el tercer factor quedó integrado por 9 ítems, y agrupó aspectos referidos a la energización y orientación del comportamiento hacia actividades que involucran las matemáticas y su aprendizaje, así como el placer o disfrute que la ejecución de tales tareas implica. Este factor fue designado gusto/motivación por las matemáticas. Las cargas factoriales de los distintos ítems se correspondieron con las dimensiones para las que habían sido originalmente seleccionados durante el desarrollo de la EAM. Las correlaciones entre los factores extraídos se expresan en la Tabla 2.
Confiabilidad de las dimensiones de la EAM

Se analizó la confiabilidad de las tres dimensiones de la EAM que fueron identificadas en el apartado anterior a través del AFE. Los resultados indicaron que los ítems que integraban cada dimensión eran consistentes entre sí: competencia percibida, $\alpha=.91$; gusto/motivación por las matemáticas, $\alpha=.89$; conducta de compromiso escolar, $\alpha=.82$.

\section{Validez predictiva}

Se contrastó la validez predictiva de la escala a través del análisis de las correlaciones entre las dimensiones de la EAM y las subescalas de productividad académica y éxito académico del APRS. Los resultados se presentan en la Tabla 3. Puede observarse que todas las correlaciones resultaron estadísticamente significativas. Las asociaciones fueron en general moderadas, con valores de $r$ oscilando entre .37 y .54 , lo que muestra la capacidad predictiva del instrumento.

\section{Discusión}

El objetivo de este estudio fue evaluar las propiedades psicométricas de la EAM para estudiantes argentinos que finalizan la escolaridad primaria e inician la secundaria y proveer evidencias sobre su validez y confiabilidad. Para el diseño de la EAM se consideraron tres dimensiones: (a) 
competencia percibida, (b) gusto/motivación por las matemáticas y (c) conductas de compromiso escolar. Estas fueron seleccionadas debido a que reflejan los tres componentes del constructo. Para las dos primeras, diferentes estudios evidenciaron que representan aspectos de los componentes cognitivo y afectivo (Adelson \& McCoach, 2011; Auzmendi, 1992; González-Pienda et al., 2012; Palacios et al., 2014; Tapia \& Marsh, 2004). Respecto a la dimensión de conductas de compromiso escolar, diferentes estudios indicaron que el esfuerzo y tiempo que los estudiantes invierten en el aprendizaje de las matemáticas afecta el desempeño y aprendizaje en esta asignatura (Fredricks et al., 2004; Hattie, 2009; Marsh et al., 2016), por lo que dicha dimensión fue desarrollada a fin de evaluar estos aspectos.

Los resultados mostraron que el cuestionario refleja la estructura propuesta de tres dimensiones y que su nivel de confiabilidad es adecuado. El primer factor, designado competencia percibida, aunó aquellos ítems que describen la percepción que los estudiantes tienen de sí mismos como aprendices de matemáticas, e incluyen descripciones de la cualidad de su desempeño y su capacidad de aprendizaje. El segundo factor, denominado conductas de compromiso escolar, agrupó los ítems que consideran el nivel de atención sobre las instrucciones y explicaciones del docente, la planificación del tiempo destinado al estudio de esta asignatura, el grado de realización de las actividades escolares, el interés por su correcta concreción y la preparación de los materiales necesarios para la clase. Por último, el tercer factor, nombrado gusto/motivación por las matemáticas, agrupó aspectos referidos a la energización y orientación del comportamiento hacia actividades que involucran las matemáticas y su aprendizaje, así como el placer o disfrute que implica la ejecución de tales tareas. Estudios previos realizados con estudiantes de nivel pri- mario y secundario de Estados Unidos (Adelson \& McCoach, 2011; Tapia \& Marsh, 2004) y España (González-Pienda et al., 2012; Palacios et al., 2014) también informaron que los ítems que describen la autopercepción del desempeño y capacidad de aprendizaje se agrupan en un factor diferente respecto de aquellos que reflejan el agrado por las actividades que involucran las matemáticas y su aprendizaje - gusto o agrado por las matemáticas- (Adelson \& McCoach, 2011; Palacios et al., 2014; Tapia \& Marsh, 2004), o de aquellos que describen la energización y activación del comportamiento hacia actividades que involucran las matemáticas -motivación- (González-Pienda et al., 2012).

Por otro lado, conforme a lo señalado en la literatura (Adelson \& McCoach, 2011; Goetz et al., 2010, 2012; Pinxten, Marsh, de Fraine, van den Noortgate, \& van Damme, 2014), el análisis de correlación entre los factores y los coeficientes de la matriz de la estructura indicaron que los factores se encuentran relacionados entre sí, siendo más fuerte el vínculo entre el factor competencia percibida y el factor gusto/motivación por las matemáticas.

Respecto de la validez predictiva de la escala, las tres subescalas propuestas mostraron correlaciones significativas, en general moderadas, con las escalas éxito académico y productividad académica de la escala APRS. La escala éxito académico mide el rendimiento del estudiante durante el trabajo escolar, su capacidad para aprender y los resultados finales del aprendizaje. Por otro lado, la escala productividad académica considera aquellos comportamientos que son importantes para alcanzar el éxito académico, tales como la realización de las actividades en clase, la capacidad de trabajo autónomo y el seguimiento de las instrucciones docentes.

Diferentes modelos teóricos sobre los factores motivacionales implicados en el aprendizaje 
académico postulan que la percepción que los estudiantes poseen respecto a su competencia dentro de un área se relaciona con el nivel de conocimiento y habilidad que alcanzan en dicha área, es decir, su éxito académico (Bandura, 1997; Connell \& Wellborn, 1990; Schunk \& Pajares, 2009). Este postulado ha sido respaldado en diversas investigaciones (Olivier, Archambault, de Clercq, \& Galand, 2019; Pajares \& Miller, 1994; Parker, Marsh, Ciarrochi, Marshall, \& Abduljabbar, 2014; Pinxten et al., 2014), por lo que la correlación significativa hallada entre la subescala competencia percibida de la EAM y la escala éxito académico del APRS aporta evidencia a favor de la validez de la primera.

Asimismo, desde el modelo del autosistema del desarrollo motivacional (Self-System Model of Motivational Development; Skinner, Furrer, Marchand, \& Kindermann, 2008; Skinner, Kindermann, Connell, \& Wellborn, 2009) se propone que las conductas de esfuerzo, dedicación e involucramiento en un área académica y las reacciones afectivas hacia ella (p. ej., entusiasmo, aprecio, interés, disfrute) predicen el desempeño y aprendizaje en dicha área académica. Las correlaciones significativas de la escala éxito académico con las escalas gusto/motivación por las matemáticas y conductas de compromiso escolar en matemáticas están en sintonía con estos postulados y evidencian su validez para la medición de tales aspectos de las actitudes hacia las matemáticas.

Por otro lado, la escala productividad académica del APRS se solapa a nivel operativo con la escala conductas de compromiso escolar, en lo que respecta a los indicadores de atención sobre las explicaciones del docente y la realización de actividades académicas. Lo anterior explicaría la relación observada entre ambas y respalda la validez de la subescala propuesta. Asimismo, distintos estudios han indicado una relación entre la cualidad y cantidad de trabajo académico (en este estudio productividad académica) y el placer o disfrute que los estudiantes experimentan durante dicha labor (Skinner et al., 2009), lo que explicaría la relación hallada entre la escala gusto por las matemáticas/ motivación y la escala productividad académica del APRS, a la vez que aporta evidencia respecto a la validez de la primera.

En síntesis, los resultados aportan evidencia sobre la confiabilidad y la validez de la EAM para la evaluación de las actitudes hacia las matemáticas en estudiantes argentinos que finalizan la escolaridad primaria e inician la secundaria. No obstante, los datos deben tomarse con cautela y futuras investigaciones deberán profundizar el estudio de las propiedades psicométricas de la EAM. Una de las limitaciones de este trabajo es que el número de participantes no ha permitido contrastar si existen diferencias en función del año escolar en los criterios de confiabilidad y validez analizados. Adicionalmente, la generalización de los resultados se encuentra obstaculizada debido al trabajo con una muestra no probabilística, que a su vez se conforma por estudiantes asistentes a una única institución educativa de gestión privada. En este sentido, será conveniente realizar nuevos estudios con muestras más numerosas y provenientes de distintos contextos socioculturales que aporten evidencia a favor a la generalización de los resultados y contrasten su estabilidad en distintos años escolares. Asimismo, futuras investigaciones deberán contrastar otras formas de validez y considerar diversos métodos de estimación de la confiabilidad del instrumento ( $p$. ej., test-retest, validez discriminante) a fin de contar con mayor evidencia sobre la confiabilidad y validez total de la escala. Pese a estas limitaciones, este trabajo representa un aporte al ofrecer una escala de medición de las actitudes hacia las matemáticas susceptible de ser aplicada a estudiantes de Argentina con evidencias de validez y confiabilidad. 


\section{Referencias}

Abal, F. J. P., Auné, S. E., \& Attorresi, H. F. (2018). Construcción y validación de una escala de actitud hacia la matemática para estudiantes de psicología. Universitas Psychologica, 17(4), 1-15. doi: 10.11144/ Javeriana.upsy17-4.cvea

Abu-Hilal, M. M. (2000). A structural model of attitudes towards school subjects, academic aspiration and achievement. Educational Psychology, 20(1), 75-84. doi: 10.1080/014434100110399

Adelson, J. L., \& McCoach, D. B. (2011). Development and psychometric properties of The Math and Me Survey. Measuring third through sixth graders' attitudes toward mathematics. Measurement and Evaluation in Counseling and Development, 44(4), 225-247. doi: 10.1177/0748175611418522

Aiken, L. R. (1974). Two scales of attitude toward mathematics. Journal for Research in Mathematics Education, 5(2), 67-71. doi: 10.2307/748616

Aiken, L. R. (1979). Attitudes toward mathematics and science in Iranian middle schools. School Science and Mathematics, 79(3), 229-234. doi: 10.1111/j.19498594.1979.tb09490.x

Aiken, L. R., \& Dreger, R. M. (1961). The effect of attitudes on performance in mathematics. Journal of Educational Psychology, 52(1), 19-24. doi: 10.1037/ h0041309

Alemany-Arrebola, I., \& Lara, A. I. (2010). Las actitudes hacia las matemáticas en el alumnado de ESO: Un instrumento para su medición. Publicaciones, 40, 49-71. Recuperado de https://revistaseug.ugr.es

American Psychological Association. (2010). Ethical principles of psychologists and code of conduct. Washington, DC: Autor. Recuperado de http://www. apa.org

Auzmendi, E. (1992). Las actitudes hacia la matemáticaestadística en las enseñanzas medias y universitaria. Características y medición. Bilbao, España: Mensajero.

Bandura, A. (1997). Self-efficacy: The exercise of control.
New York, NY: W. H. Freeman.

Bazán, J. L., \& Sotero, H. (1998). Una aplicación al estudio de actitudes hacia la matemática en la UNALM. Anales Científicos UNALM, 36, 60-72. Recuperado de http://argos.pucp.edu.pe/ jlbazan

Burrus, J., \& Moore, R. (2016). The incremental validity of beliefs and attitudes for predicting mathematics achievement. Learning and Individual Differences, 50, 246-251. doi: 10.1016/j.lindif.2016.08.019

Butterworth, B. (2005). The development of arithmetical abilities. Journal of Child Psychology and Psychiatry, 46(1), 3-18. doi: 10.1111/j.1469-7610.2004.00374.x

Cardoso-Espinosa, E. O., Vanegas-López, E. A., \& Cerecedo-Mercado, M. T. (2012). Diagnóstico sobre las actitudes hacia las matemáticas del estudiantado que inicia sus estudios en tres posgrados en Administración de Empresas. Revista Electrónica EDUCARE, 16(2), 237-253. doi: 10.15359/ree.162.15

Chambers, C. T., \& Johnston, C. (2002). Developmental differences in children's use of rating scales. Journal of Pediatric Psychology, 27(1), 27-36. doi: 10.1093/ jpepsy/27.1.27

Chen, L., Bae, S. R., Battista, C., Qin, S., Chen, T., Evans, T. M., \& Menon, V. (2018). Positive attitude toward math supports early academic success: Behavioral evidence and neurocognitive mechanisms. Psychological Science, 29(3), 390-402. doi: $10.1177 / 0956797617735528$

Connell, J. P., \& Wellborn, J. G. (1990). Competence, autonomy, and relatedness: A motivational analysis of self-system process. En M. R. Gunnar \& L. A. Sroufe (Eds.), Self-processes and development. The Minnesota Symposia on Child Development (pp. 43-77). Hillsdale, NJ: Lawrence.

Cubillo, C., \& Ortega, T. (2000). Influencia de un modelo didáctico en la opinión/actitud de los alumnos hacia las matemáticas. RELIME. Revista Latinoamericana de Investigación en Matemática Educativa, 3(2), 189-206. Recuperado de https://relime.org

Cueli, M., González-Castro, P., Álvarez, L., García, T., \& 
González-Pienda, J. A. (2014). Variables afectivomotivacionales y rendimiento en matemáticas: Un análisis bidireccional. Revista Mexicana de Psicología, 31(2), 153-163. Recuperado de http:// comeppsi.com/rmp

Deci, E. L., \& Ryan, R. M. (1985). Intrinsic Motivation and Self-determination in Human Behavior. New York, NY: Springer. doi: 10.1007/978-1-4899-2271-7

Deci, E. L., \& Ryan, R. M. (2000). The "what" and "why" of goal pursuits: Human needs and the self determination of behavior. Psychological Inquiry, 11(4), 227-268. doi: 10.1207/S15327965PLI1104_01

Dörfer, C., \& Ulloa-Duque, G. S. (2016). Medición de la actitud hacia las matemáticas en estudiantes de Licenciatura en Administración: Un estudio piloto. VinculaTégica. EFAN, 2(1), 1329-1348. Recuperado de http://www.web.facpya.uanl.mx/vinculategica/

DuPaul, G. J., Rapport, M. D., \& Perriello, L. M. (1991). Teacher ratings of academic skills: The development of the academic performance rating scale. School Psychology Review, 20(2), 284-300. Recuperado de https://www.tandfonline.com

Eagly, A., \& Chaiken, S. (2005). Attitude research in the 21st century: The current state of knowledge. En D. Albarracín, B. T. Johnson \& M. P. Zanna (Eds.), The Handbook of Attitudes (pp. 743-767). Mahwah, NJ: Lawrence Erlbaum.

Eccles, J. S., \& Wigfield, A. (1995). In the mind of the actor: The structure of adolescents' achievement task values and expectancy-related beliefs. Personality and Social Psychology Bulletin, 21(3), 215-225. doi: $10.1177 / 0146167295213003$

Eccles, J. S., \& Wigfield, A. (2002). Motivational beliefs, values, and goals. Annual Review of Psychology, 53(1), 109-132. doi: 10.1146/annurev. psych.53.100901.135153

Eccles, J., Wigfield, A., Harold, R. D., \& Blumenfeld, P. (1993). Age and gender differences in children's selfand task perceptions during elementary school. Child Development, 64(3), 830-847. doi: 10.2307/1131221 Escalera-Chávez, M. E., Moreno-García, E., \& Ro-
jas-Kramer, C. A. (2019). Confirmatory model to measure attitude towards mathematics in higher education students: Study case in SLP Mexico. International Electronic Journal of Mathematics Education, 14(1), 163-168. doi: 10.29333/iejme/3984

Farías-Mata, M. A. (2011). Actitudes y autorregulación en el aprendizaje de la matemática. Nuevos abordajes en la enseñanza-aprendizaje de la matemática en estudiantes universitarios. Alemania: Académica Española.

Farías-Mata, M. A. (2015). Revalidación psicométrica del cuestionario de actitudes hacia la matemática en estudiantes universitarios. Revista Evaluar, 15(1), 75-98. Recuperado de https://revistas.unc.edu.ar

Fennema, E., \& Sherman, J. A. (1976). Fennema-Sherman Mathematics Attitudes Scales: Instruments designed to measure attitudes toward the learning of mathematics by females and males. Journal for Research in Mathematics Education, 7(5), 324-326. doi: $10.2307 / 748467$

Fredricks, J. A., Blumenfeld, P. C., \& Paris, A. H. (2004). School engagement: Potential of the concept, state of the evidence. Review of Educational Research, 74(1), 59-109. doi: 10.3102/00346543074001059

Fredricks, J. A., Wang, M. T., Schall-Linn, J., Hofkens, T. L., Sung, H. C., Parr, A. K., \& Allerton, J. J. (2016). Using qualitative methods to develop a survey measure of math and science engagement. Learning and Instruction, 43, 5-15. doi: 10.1016/j.learninstruc.2016.01.009

Goetz, T., Cronjäger, H., Frenzel, A. C., Lüdtke, O., \& Hall, N. C. (2010). Academic self-concept and emotion relations: Domain specificity and age effects. Contemporary Educational Psychology, 35(1), 44-58. doi: 10.1016/j.cedpsych.2009.10.001

Goetz, T., Nett, U. E., Martiny, S. E., Hall, N. C., Pekrun, R., Dettmers, S., \& Trautwein, U. (2012). Students' emotions during homework: Structures, self-concept antecedents, and achievement outcomes. Learning and Individual Differences, 22(2), 225-234. doi: 10.1016/j.lindif.2011.04.006 
Gómez-Chacón, I. M. (2009). Actitudes matemáticas: Propuestas para la transición del bachillerato a la universidad. Educación Matemática, 21(3), 5-32. Recuperado de https://www.revista-educacionmatematica.org.mx

González-Pienda, J. A., Fernández-Cueli, M., García, T., Suárez, N., Fernández, E., Tuero-Herrero, E., \& da Silva, E. H. (2012). Diferencias de género en actitudes hacia las matemáticas en la enseñanza obligatoria. Revista Iberoamericana de Psicología y Salud, 3(1), 55-73. Recuperado de https:/www. redalyc.org/pdf/2451/245122736004.pdf

Guvercin, S., \& Verbovskiy, V. (2014). The effect of problem posing tasks used in mathematics instruction to mathematics academic achievement and attitudes toward mathematics. International Online Journal of Primary Education (IOJPE), 3(2), 59-65. Recuperado de http://www.iojpe.org

Hattie, J. (2009). Visible learning. A synthesis of over 800 meta-analyses relating to achievement. Londres, Reino Unido: Routledge.

Hemmings, B., Grootenboer, P., \& Kay, R. (2011). Predicting mathematics achievement: The influence of prior achievement and attitudes. International Journal of Science and Mathematics Education, 9(3), 691-705. doi: 10.1007/s10763-010-9224-5

Hurtado-Mondoñedo, L. (2011). Validación de una escala de actitudes hacia las matemáticas. Investigación Educativa, 15(28), 99-108. Recuperado de https:// revistasinvestigacion.unmsm.edu.pe/

Lozano, L. M., García-Cueto, E., \& Muñiz, J. (2008). Effect of the number of response categories on the reliability and validity of rating scales. Methodology: European Journal of Research Methods for the Behavioral and Social Sciences, 4(2), 73-79. doi: 10.1027/1614-2241.4.2.73

Marsh, H. W., Pekrun, R., Lichtenfeld, S., Guo, J., Arens, A. K., \& Murayama, K. (2016). Supplemental material for breaking the double-edged sword of effort/ trying hard: Developmental equilibrium and longitudinal relations among effort, achievement, and academic self-concept. Developmental Psychology, 52(8), 1273-1290. doi: 10.1037/dev0000146.supp

Melancon, J. G., Thompson, B., \& Becnel, S. (1994). Measurement integrity of scores from the Fennema-Sherman Mathematics Attitudes Scales: The attitudes of public school teachers. Educational and Psychological Measurement, 54(1), 187-192. doi: 10.1177/0013164494054001024

Mellor, D., \& Moore, K. A. (2014). The use of likert scales with children. Journal of Pediatric Psychology, 39(3), 369-379. doi: 10.1093/jpepsy/jst079

Meza-Cascante, L. G., Agüero-Calvo, E., Suárez-ValdésAyala, Z., Calderón-Ferrey, M., Sancho-Martínez, L., Pérez-Tyteca, P., \& Monje Parrilla, J. (2019). Actitud hacia la matemática: Percepción de la actitud de padres. Comunicación, 28(1), 4-15. doi: 10.18845/ rc.v28i1-2019.4437

Ministerio de Educación y Deportes. (2017). Aprender 2016. Informe de resultados. Recuperado de http:// www.bnm.me.gov.ar/giga1/documentos/EL005597. pdf

Ministerio de Educación, Cultura, Ciencia y Tecnología. (2019). Aprender 2018. Informe nacional de resultados. $6^{\circ}$ año nivel primario. Recuperado de http://www.bnm.me.gov.ar/giga1/documentos/ EL006420.pdf

Miñano, P., \& Castejón, J. L. (2011). Variables cognitivas y motivacionales en el rendimiento académico en lengua y matemáticas: Un modelo estructural. Revista de Psicodidáctica, 16(2), 203-230. Recuperado de https://www.ehu.es

Miranda, A. (2012). Funcionamiento ejecutivo y motivación en tareas de cálculo y solución de problemas de niños con TDAH. Revista de Psicodidáctica, 17(1), 51-72. Recuperado de https://www.ehu.es

Montero, I., \& León, O. G. (2005). Sistema de clasificación del método en los informes de investigación en psicología. International Journal of Clinical and Health Psychology, 7(3), 847-862. Recuperado de http://www.aepc.es

Mulhern, F., \& Rae, G. (1998). Development of a short- 
ened form of the Fennema-Sherman Mathematics Attitudes Scales. Educational and Psychological Measurement, 58(2), 295-306. doi: 10.1177/0013164498058002012

Muñoz-Cantero, J. M., \& Mato-Vázquez, M. D. (2006). Diseño y validación de un cuestionario para medir las actitudes hacia las matemáticas en alumnos de ESO. Revista Galego-Portuguesa de Psicoloxía e Educación, 13(11-12), 413-424. Recuperado de https://ruc.udc.es

Muñoz-Cantero, J. M., \& Mato-Vázquez, M. D. (2008). Análisis de las actitudes respecto a las matemáticas en alumnos de ESO. Revista de Investigación Educativa, 26(1), 209-226. Recuperado de http:// revistas.um.es

Núñez, J. C., González-Pienda, J. A., Alvarez, L., González, P., González-Pumariega, S., Roces, C., ... Rodrigues-Feio, L. do S. (2005). Las actitudes hacia las matemáticas: Perspectiva evolutiva. Actas do VIII Congreso Galaico-Portugués de Psicopedagoxía (pp. 2389-2396). Recuperado de http://www. educacion.udc.es

O’Neal, M. R., Ernest, P. S., McLean, J. E., \& Templeton, S. M. (Noviembre, 1988). Factorial validity of the Fennema-Sherman Mathematics Attitudes Scales. Trabajo presentado en The Annual Meeting of the Mid-South Educational Research Association, Louisville, KY. (ERIC Document Reproduction Service ED303493). Recuperado de https://eric.ed.gov

Olivier, E., Archambault, I., De Clercq, M., \& Galand, B. (2019). Student self-efficacy, classroom engagement, and academic achievement: Comparing three theoretical frameworks. Journal of Youth and Adolescence, 48(2), 326-340. doi: 10.1007/s10964-0180952-0

Organisation for Economic Cooperation and Development. (2019). PISA 2018 Results. What Students Know and Can Do (Vol. I). París, Francia: Autor. doi: $10.1787 / 5 \mathrm{f0} 7 \mathrm{c} 754-\mathrm{en}$

Pajares, F., \& Miller, M. D. (1994). Role of self-efficacy and self-concept beliefs in mathematical problem solving: A path analysis. Journal of Educational Psychology, 86(2), 193-203. doi: 10.1037/00220663.86.2.193

Palacios, A., Arias, V., \& Arias, B. (2014). Las actitudes hacia las matemáticas: Construcción y validación de un instrumento para su medida. Revista de Psicodidáctica, 19(1), 67-91. doi: 10.1387/ RevPsicodidact.8961

Parker, P. D., Marsh, H. W., Ciarrochi, J., Marshall, S., \& Abduljabbar, A. S. (2014). Juxtaposing math self-efficacy and self-concept as predictors of long-term achievement outcomes. Educational Psychology, 34(1), 29-48. doi: 10.1080/01443410.2013.797339

Pedrosa, M. E., Astiz, M., Montero, Y., \& Todisco, N. (Junio, 2016). Elaboración y análisis de una escala para el estudio de las actitudes de los alumnos de la secundaria superior hacia la matemática. En Actas de las III Jornadas de Investigadores en Educación. Facultad de Humanidades, Universidad Nacional de Mar del Plata, Argentina. Recuperado de https:// fh.mdp.edu.ar

Perels, F., Dignath, C., \& Schmitz, B. (2009). Is it possible to improve mathematical achievement by means of self-regulation strategies? Evaluation of an intervention in regular math classes. European Journal of Psychology of Education, 24(1), 17-31. doi: 10.1007/ BF03173472

Petriz-Mayen, M. A., Barona-Ríos, C., López-Villarreal, R. M., \& Quiroz-González, J. (2010). Niveles de desempeño y actitudes hacia las matemáticas en estudiantes de la Licenciatura en Administración en una universidad estatal mexicana. Revista Mexicana de Investigación Educativa, 15(47), 1223-1249. Recuperado de http://www.scielo.org.mx/pdf/rmie/ v15n47/v15n47a12.pdf

Pinxten, M., Marsh, H. W., de Fraine, B., van den Noortgate, W., \& van Damme, J. (2014). Enjoying mathematics or feeling competent in mathematics? Reciprocal effects on mathematics achievement and perceived math effort expenditure. British Journal of Educational Psychology, 84(1), 152-174. doi: 10.1111/ 
bjep. 12028

Pitsia, V., Biggart, A., \& Karakolidis, A. (2017). The role of students' self-beliefs, motivation and attitudes in predicting mathematics achievement: A multilevel analysis of the Programme for International Student Assessment data. Learning and Individual Differences, 55, 163-173. doi: 10.1016/j.lindif.2017.03.014

Qaisar, S., Dilshad, M., \& Butt, I. H. (2015). Influence of collaborative group work on students' attitude towards mathematics. Journal of Educational Research, 18(1), 69-84. Recuperado de http://jer.iub. edu.pk

Román-Alegre, B., Mera-Cantillo, C., Aragón-Mendizábal, E., \& Delgado, C. (2019). Descripción de una escala de actitudes hacia la matemática temprana (ESAMAT). Revista INFAD de Psicología. International Journal of Developmental and Educational Psychology, 3(1), 213-220. doi: 10.17060/ijodaep.2019.n1.v3.1472

Russell, D. W. (2002). In search of underlying dimensions: The use (and abuse) of factor analysis in Personality and Social Psychology Bulletin. Personality and Social Psychology Bulletin, 28(12), 1629-1646. doi: 10.1177/014616702237645

Ryan, R. M., \& Deci, E. L. (2000). Intrinsic and extrinsic motivations: Classic definitions and new directions. Contemporary Educational Psychology, 25(1), 5467. doi: 10.1006/ceps.1999.1020

Schunk, D. H., \& Pajares, F. (2009). Self-efficacy theory. En K. R. Wentzel \& A. Wigfield (Eds.), Handbook of motivation at school (pp. 35-54). New York, NY: Routledge. doi: 10.4324/9780203879498

Skinner, E. A., Furrer, C., Marchand, G., \& Kindermann, T. A. (2008). Engagement and disaffection in the classroom: Part of a larger motivational dynamic? Journal of Educational Psychology, 100(4), 765-781. doi: $10.1037 / \mathrm{a} 0012840$

Skinner, E. A., Kindermann, T. A., Connell, J. P., \& Wellborn, J. G. (2009). Engagement and disaffection as organizational constructs in the dynamics of motivational development. En K. R. Wentzel \& A. Wigfield
(Eds.), Handbook of Motivation at School (pp. 223245). New York, NY: Routledge.

Stelzer, F., Canet-Juric, L., Andrés, M. L., Vernucci, S., \& Richards, M. (Agosto, 2019). Estudio preliminar de confiabilidad y validez convergente y predictiva de la escala "brief-2 familia” para la medición de la memoria de trabajo, inhibición y flexibilidad en niños de Argentina. Póster presentado en la XVII Reunión Nacional y VI Encuentro Internacional de la AACC. Posadas, Misiones, Argentina.

Stipek, D. (2002). Good instruction is motivating. En A. Wigfield \& J. S. Eccles (Eds.), Development of achievement motivation (pp. 309-332). San Diego, CA: Academic Press.

Suinn, R. M., \& Edwards, R. (1982). The measurement of mathematics anxiety: The Mathematics Anxiety Rating Scale for Adolescents-MARS A. Journal of Clinical Psychology, 38(3), 576-580.

Tapia, M., \& Marsh, G. E. (2004). An instrument to measure mathematics attitudes. Academic Exchange Quarterly, 8(2). Recuperado de http://www.rapidintellect.com/

Vallejo-Seco, G., \& Escudero-García, J. R. (1999). Cuestionario para evaluar las actitudes de los estudiantes de E.S.O. hacia las Matemáticas. Aula Abierta, 26(74), 193-208. Recuperado de https://www.unioviedo.es/reunido/index.php/AA/index

Vázquez-Alonso, A., \& Manassero-Mas, M. A. (1995). Actitudes relacionadas con la ciencia: Una revisión conceptual. Enseñanza de las ciencias: Revista de Investigación y Experiencias Didácticas, 13(3), 337346. Recuperado de: https://ddd.uab.cat

Watt, H. M. G. (2000). Measuring attitudinal change in mathematics and english over 1st year of junior high school: A multidimensional analysis. The Journal of Experimental Education, 68(4), 331-361. doi: 10.1080/00220970009600642

Wieland, A., Durach, C. F., Kembro, J., \& Treiblmaier, H. (2017). Statistical and judgmental criteria for scale purification. Supply Chain Management, 22(4), 321328. doi: 10.1108/SCM-07-2016-0230 
World Medical Association. (2013). Declaration of Helsin$\mathrm{ki}$-Ethical principles for medical research involving human subjects. Recuperado de https://www.wma. net/policies-post/wma-declaration-of-helsinki-ethical-principles-for-medical-research-involving-human-subjects/

Zaichkowsky, J. L. (1994). The Personal Involvement Inventory: Reduction, revision, and application to advertising. Journal of Advertising, 23(4), 59-70. doi: 10.1080/00913367.1943.10673459

Zanna, M., \& Rempel, J. (1988). Attitudes: A new look at an old concept. En D. Bar-Tal \& A. Kruglanski (Eds.), The Social Psychology of Knowledge (pp. 315-334). Cambridge, Reino Unido: Cambridge University. dio $\quad 10.1002 / 1097-4679(198207) 38: 3<576:: a i d-$ jclp2270380317>3.0.co;2-v 